\title{
COMPUTATIONAL ASPECTS OF TRANSIENT FIELDS ASSOCIATED WITH INSULATION BREAKDOWN IN G I S UNDER INHOMOGENEOUS FIELDS
}

\author{
H. N. Suresh* \\ M. S. Naidu \\ Department of High Voltage Engineering \\ Indian Institute of Science \\ Bangalore - 560 012, INDIA \\ E-mail: suresh@hve.iisc.ernet.in \\ msnaidu@hve.iisc.ernet.in
}

\begin{abstract}
The phenomena of leader inception and propagation leading to breakdown in strongly attaching gases like $\mathrm{SF}_{6}$ are currently being studied because of their relevance to the design of High Voltage Gas Insulated Systems (GIS). Breakdown of $\mathrm{SF}_{6}$ gas under inhomogeneous fields is known to be by the leader mechanism. The leader propagation is guided by the electric field at the leader tip, which inturn is dependent on the nature of voltage applied, electrode geometry and charge distributed in the leader. The charge build up process in the leader is governed by ResistiveCapacitive (R-C) transient fields. In such fields, both permittivities and conductivities of the participating medium determine the field distribution. The scope of the present paper is to discuss the important aspects of computing R-C transient field associated with the leader in $\mathrm{SF}_{6}$ gas modeled as a straight cylindrical conducting channel. Studies are carried out under unit step voltage excitation for two leader lengths and assumed conductivity of the leader. The workability of the R-C transient field computing algorithm for such studies carried out with the help of a Laplace solver are presented and discussed.
\end{abstract}

\section{Introduction}

Excellent insulating properties of $\mathrm{SF}_{6}$ gas have made it to be a popular dielectric in High Voltage apparatus like GIS. In a GIS, vast majority of the power components are safely sealed in a clean environment of dry $\mathrm{SF}_{6}$ gas. During the course of their operation, GIS are subjected to Very Fast Transient (VFT) over voltages which quite often are found to cause the $\mathrm{SF}_{6}$ insulation failure. Experimental investigations in this

* Senior Lecturer, Malnad College of Engineering, Hassan - 573 201, Karnataka. Currently, a Research student at Indian Institute of Science, Bangalore - 560012. regard have suggested that the breakdown of insulation is by the leader mechanism. Attempts have been made in the recent past to macroscopically model the leader growth process[1-6]. Earlier works in this area have employed a lumped R-C circuit model in which streamer is represented by Resistance and Capacitance in parallel. Electrostatic coupling to ground is approximated by a single capacitance connecting streamer head to ground. With the lumped circuit model of that kind, it is not possible to account for the electrostatic coupling of leader and streamer with each other and with respect to the ground. A distributed circuit approach looks like an alternative. However, even with the distributed circuit approach, it is nearly impossible to compute the various capacitances involved. Therefore, it would be appropriate to employ field theoretical approach to such studies.

\section{Modeling}

The discharge development in case of inhomogeneous fields leading to breakdown of $\mathrm{SF}_{6}$ insulation occurs by the leader mechanism typically at pressures above 0.5 bar[1]. Therefore, field approach to Modeling the $\mathrm{SF}_{6}$ insulation breakdown under inhomogeneous fields involves (i) Modeling the leader mechanism and (ii) Computation of the associated transient field. In this paper, the second part of computing transient field has been discussed in particular.

The electrode geometry chosen consisting of a high voltage hemispherically tipped electrode and a grounded plane electrode is shown in Figure 1. The electrostatic field in the electrode gap is governed by the Laplace equation

$$
\nabla^{2} \phi=0
$$

This basically necessiates the development of an accurate electric field calculation tool in the form of a Laplace solver. The Laplace solver used has been developed from the 
fundamental principles of Charge Simulation Method (CSM)[7].The high voltage electrode is modeled using ring charges over major portion of the high voltage electrode and a point charge in its hemispherical tip portion. The plane electrode is simulated by taking into account the image charges. The field computations were initially carried out in the bare gap (in the absence of any leader discharge) to asses the field non-uniformity factor. The non-uniformity factor calculation for the given gap was cross checked with the empirical equations suggested in the literature[8].

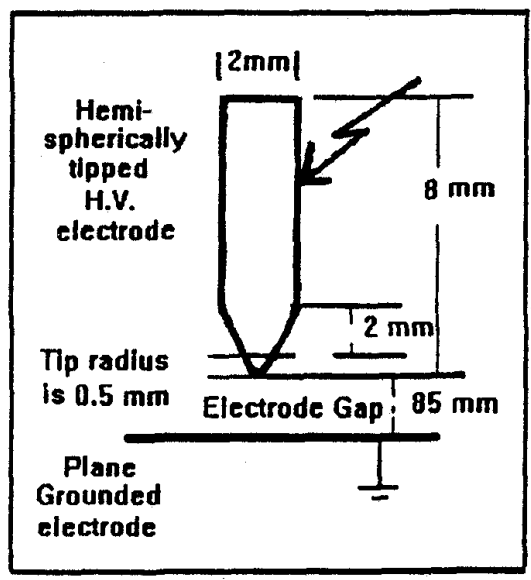

Figure 1. Electrode Geometry

Transient field computational studies associated with the leader propagation process involve modeling the leader channel and computation of associated R-C transient field. The leader originating at the electrode tip, once the critical field gradient $\left(89.6 \mathrm{kV} / \mathrm{cm}\right.$-bar in $\left.\mathrm{SF}_{6}\right)$ is established is modeled using cylindrical charges placed axially. With the assumption of a vertical leader, the problem becomes axisymmetric and hence the discretisation needs to be carried out along the axis of the high voltage electrode only. The leader is sectionalised into definite number of cylindrical charge segments each with linearly varying charge density. In $\mathrm{z}$-direction the variation in charge density for each segment is

$$
\lambda=\lambda_{2}\left(\mathbf{z}-\mathbf{z}_{1}\right) /\left(\mathbf{z}_{2}-\mathbf{z}_{1}\right)+\lambda_{1}\left(\mathbf{z}_{2}-\mathbf{z}\right) /\left(\mathbf{z}_{2}-\mathbf{z}_{1}\right) \ldots \ldots
$$

The boundary conditions that are valid at these leader segments are

$$
E_{1 t}=E_{2 t}
$$

$$
\begin{gathered}
\mathbf{D}_{\mathbf{n} \mathbf{1}}-\mathbf{D}_{\mathbf{n} \mathbf{2}}=\sigma_{\mathbf{s}} \\
\mathbf{J}_{\mathbf{1 n}}-\mathbf{J}_{\mathbf{2 n}}+\nabla_{\mathbf{s}} \cdot \mathbf{J}_{\mathbf{s}}=-\partial \sigma_{\mathbf{s}} / \partial t
\end{gathered}
$$

The boundary condition (5) governs the time dependence of the field quantities. It suggests that at any instant, difference in normal components of conduction current densities and divergence of surface current density of the leader will be compensated by the displacement current densities. Such class of fields are classified as Resistive-Capacitive (R-C) fields.

One may appreciate that it is necessary to cross check the authenticity of electric field calculation routines used to evaluate the potential and electric field at the tip of each of these segments. In order to achieve this, the electric field gradients of these leader segments were initially deliberately fixed. Then tip potential/electric field gradients at the end of each of these segments were computed. The highest error in such tip gradient computation against the assigned gradient was $0.02 \%$.

The R-C transient algorithm mentioned in the next section is then applied to this leader to study the temporal variation of leader tip potential under a unit step voltage applied to the High Voltage electrode.The studies are carried out for the two distinct extreme cases of leader extention in space. viz. $10 \%$ and $98 \%$ of the gap from high voltage electrode. Typical leader current density and average electric field of the leader noticed in the literature[ $1 \& 3]$ are $50 \mathrm{~A} / \mathrm{cm}^{2}$ and 3 $\mathrm{kV} / \mathrm{cm}$ respectively. Assuming a leader radius of $220 \mu \mathrm{m}$ and using the above typical values, the conductivity of the leader was fixed to be $1.667 \mathrm{v} / \mathrm{m}$.

\section{Algorithm}

The R-C transient algorithm has been successfully employed for field transient studies[9] and is proved to be very useful. Under the specific excitation, with the given boundary conditions, the steps followed are:

1. At zero time step $t_{0}$, the problem is solved as an electrostatic one i.e., by giving the permittivity and initial charge distribution (if any) as inputs to the Poisson's solver. The resulting current is assumed to be approximately constant over a short interval of time $\Delta t$. With this assumption, charge accumulated in that interval is computed using the relation

$$
\lambda_{z}(t+\Delta t)=\lambda_{z}(t) \cdot \Delta t\left[\partial \mathbf{J}_{z} / \partial \mathbf{z}\right]
$$


The charge computed is used in the field calculation in the next time step.

2. The charge densities computed for the time steps $t_{n}$ from the time steps $t_{n-1}$ are used for the field calculation (as explained in step 1) at time steps $t_{n}(n=0,1,2,3 \ldots$.$) .$

3. Using the field computed at time step $t_{n}$ the charge accumulated till time $t_{n+1}$ is computed using the equation in step 1.

4. Steps 2 and 3 are repeated to cover the desired time duration.

\section{$\underline{\text { Results and Discussion }}$}

Using the model and algorithm described in the earlier sections, the computation of the leader tip potential is made. The nature of build up of potential at the tip of leader for a leader length equal to $10 \%$ and $98 \%$ of the gap from the high voltage electrode are shown in figures $2 \& 3$. There is a smooth build up of leader tip potential (see Figures $2 \& 3$ ) which finally settles to the assigned magnitude of the step voltage excitation applied to the high voltage electrode.

The observations made during computation of the transient field are as follows:

For obtaining good accuracy and temporal resolution, it is necessary to have small time steps. This will be at the cost of larger computational time and storage. An approximate time step is to be initially chosen and a study has to be carried out with one small and one large time step. The signs of the interface charge densities computed are compared. Sign reversal takes place only if the second time step chosen is large. By trial and error, the second time step chosen is reduced to obtain no sign change, thereby getting an idea of the time step to be taken.

It is also worth noting that digital filtering technique[10] has been incorporated to reduce the numerical oscillations of computed field quantities. The maximum time step that could have been selected in this particular study are 1.0e-06 sec while filter was employed and $1.0 \mathrm{e}-08 \mathrm{sec}$ in the absence of filter. Eventhough, the results obtained with and without filter in figures $2 \& 3$ are aligning, one should note that maximum time step that could be chosen under the usage of filter is two order high (without causing instability of computed quantity) in comparison to that of without filter.
Higher the time step chosen, lower is the computational time required. Thus, use of filter also brings down the computational time by a good order. Smooth build up of potential and field at the leader tip with or without the inclusion of the filter is indicative of the versatility of the algorithm.

\section{Conclusions}

In summary,

1. The suggested model for the leader in this paper along with the R-C transient algorithm efficiently traces the field quantities at the leader tip over space and time.

2.The incorporated technique is inherently complete in the sense that leader head to ground capacitance is not considered as lumped equivalent for the leader sections. The suggested field approach doesnot give room to the omission of any of the associated capacitances.

3.The filtering algorithm employed would help in the suppression of the numerical oscillations in the computation of the leader quantities. Using the filter, a higher time step can be employed without causing numerical instability in the computation. This would bring down the total computational time.

\section{$\underline{\text { References }}$}

[1] I.D. Chalmers, I. Gallimberti, A. Gibert and O. Farish, "The development of electrical leader discharges in a Pointplane gap in $S_{6}$ ", Proc. Royal Soc. London, A412, pp 285 308, 1987.

[2] Heinrich Hiesinger, "The calculation of leader propagation in Point/plane gaps under very fast transient stress", Proc. VI Int. Symposium on Gaseous Dielectrics, pp $129-135,1991$.

[3] L. Niemeyer, L. Ullrich and N. Wiegart, "The mechanism of leader breakdown in electronegative gases", IEEE Transactions on EI, Vol. 24, No.2, pp309 -324, April 1989.

[4] B. Heers, "Leader propagation in inhomogeneous $S F_{6}$ gaps under VFT stress with critical frequencies", Ninth International Symposium on High Voltage Engineering, Austria, pp 2267-1 to 2267-4, 1995. 
[5] Dietmar Buchner, "Discharge development in $\mathrm{SF}_{6}$ in case of composite voltage stress", Gaseous Dielectrics VII, Ed. by L.G. Christophorou and D.R. James, Plenium Press, New York, pp 291 -297, 1994.

[6] K. Tekletsadik, L.C. Campbell, "SF 6 breakdown in GIS", IEE proceedings - Science, Measurement, Technology, Vol. 143, No. 5, pp 270 - 276, Sept. 1996.

[7] Nazar H. Malik, "A Review of the Charge Simulation Method and its applications", IEEE Transactions on EI, Vol. 24, No. 1, pp 3 - 20, Feb. 1989.

[8] Y. Qiu, "Simple expression of field Non-uniformity factor for Hemispherically capped Rod-plane gaps", IEEE Transactions on EI, Vol. 21, No. 4, pp 673 - 675, August 1986.

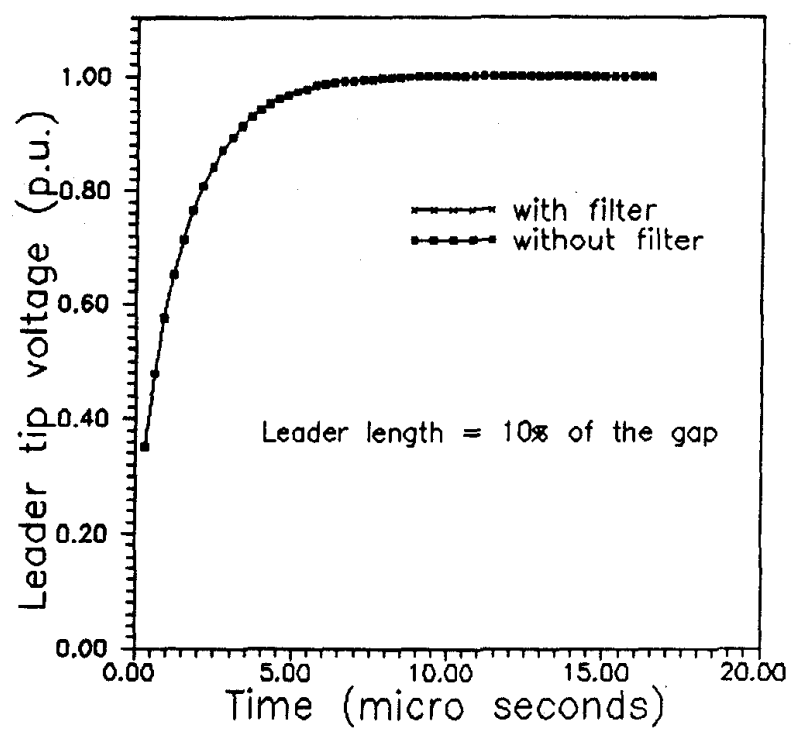

Figure 2. Temporal build up of Leader tip potential
[9] Udaya Kumar, G.R. Nagabhushana, "Solution of Capacitive-Resistive transient fields", Ninth Inter-national symposium on High Voltage Engineering, Austria, pp 83511 to $8351-4,1995$.

[10] W. Shyy, M. H. chen, R. Mittal and H. S. Udaya Kumar, "On the suppression of Numerical oscillations using a Non-linear filter", Journal of Computational Physics, Vol. 102, pp $49-62,1992$.

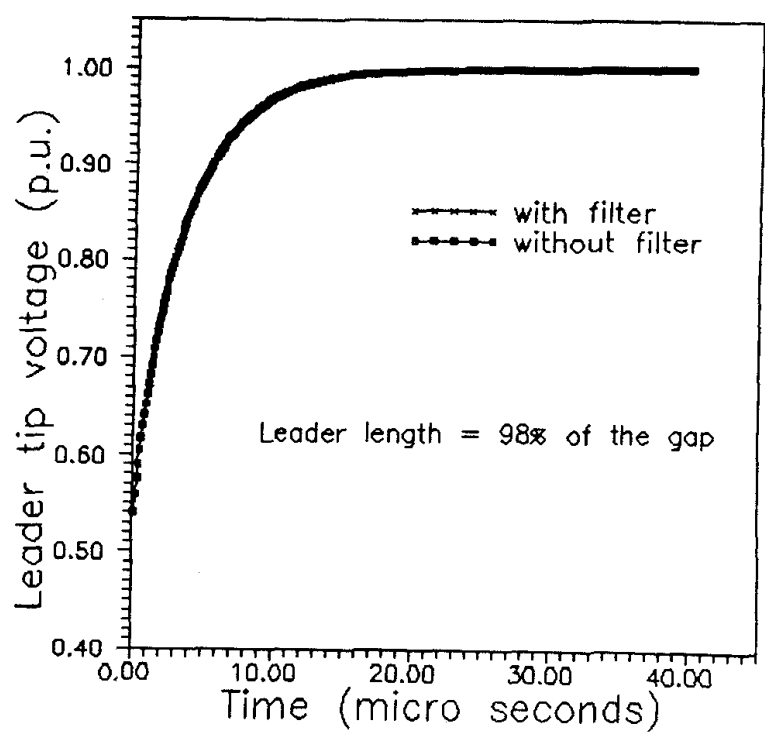

Figure 3. Temporal build up of Leader tip potential 